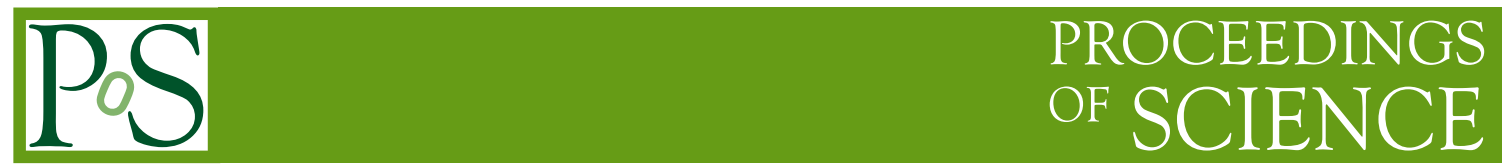

\title{
Report on outreach activities at the LHC
}

\author{
Despina Hatzifotiadou ${ }^{a, 1, *}$ \\ ${ }^{a}$ INFN Bologna, Italy and EP Department, CERN \\ E-mail: despina.hatzifotiadou@cern.ch
}

Outreach is undoubtedly an integral part of the scientific process. Reaching out to the public, explaining our results and sharing our enthusiasm, we try to motivate the youth, to clarify misconceptions about science and to ensure continued funding - to name but a few of our goals. The LHC community is very active in this domain, organising various events and using a variety of methods and tools. A selection of outreach and educational activities coordinated and supported by the LHC collaborations is presented.

The Eighth Annual Conference on Large Hadron Collider Physics-LHCP2020

25-30 May, 2020

online

${ }^{1}$ on behalf of ALICE, ATLAS, CMS and LHCb collaborations

* Speaker 


\section{Introduction}

Outreach and communication are undeniably an integral part of the scientific process. Our target groups are diverse, and so are the aims we try to achieve: explain to the general public what we do and how we do it and thus increase their scientific literacy and demystify science; tell the taxpayers how their money is invested for research; inspire the youth and prepare the next generation of scientists; inform the media, who have great power over the public; convince decision makers about the necessity of fundamental research and thus ensure funding; provide evidence-based information, since plenty of wrong facts and conspiracy theories circulate on the web.

Out of the numerous activities of the LHC experiments' community to meet these goals, a personal selection is presented here, since limitation in space makes it impossible to give credit to all.

\section{CERN and the LHC Collaborations}

The CERN ECO (Education, Communications and Outreach) group organises a multitude of educational programmes and other activities: teachers programmes, internships for high-school students, visits, open days, exhibitions, production of audiovisual material - to name but a few. The LHC collaborations contribute to CERN's efforts participating in these activities. They also organise their own events, produce educational material and have various channels for communication and outreach. Individual members, institutes and countries of the LHC collaborations also develop their own outreach and educational projects. Each LHC experiment has an outreach coordinator or coordination group. At CERN, informal LOG (LHC Outreach Group) meetings are held regularly, to facilitate exchange of information, share experiences and coordinate common activities.

\section{IPPOG}

Soon after the establishment of EPPOG, the European Particle Physics Outreach Group, in 1997, the LHC experiments had become members. In 2011, having expanded beyond Europe, the group changed its name to IPPOG (International Particle Physics Outreach Group). IPPOG [1], a network of scientists, science educators and communication specialists promoting informal science education and particle-physics outreach across the globe, became an International Collaboration in 2016. Nowadays it has 33 members (26 countries, 6 experiments and CERN); the four big LHC experiments have signed MoUs specifying that they offer in-kind contribution, such as data and software tools for the masterclasses.

\section{International Masterclasses in Particle Physics}

The flagship activity of IPPOG is the International Masterclasses in Particle Physics [2], a very successful hands-on educational programme for high-school students, organised every year for a period of six weeks, during February, March and April. Students are invited to institutes and are initiated to the world of scientific research by becoming physicists for a day. After introductory talks they analyse, using dedicated software tools, real data from an experiment leading to a physics 
measurement. At the end of the day, during a videoconference with other institutes, they present their results and discuss further, as it is done by international collaborations. The main bulk of the international masterclasses is done with data from the four big LHC experiments. In an effort to expand the scope of the masterclasses, more experiments have recently proposed exercises; a particle-therapy masterclass has also been developed, introducing students to applications of our field for society. This programme has been growing spectacularly over the years and last year (2019) 15000 students and 1000 teachers participated in masterclasses held in 54 countries.

\section{Open Data and LHC@home}

Going a step further, the LHC experiments have released data - much more than what is used for the masterclasses - as well as the tools for their analysis, for public use, on the CERN Open Data portal [3]. The open data are mainly aimed at university students, but also for anybody with an interest and motivation. For many educational examples with LHC Open Data see Ref. [4]. Another example of the public's involvement with LHC data/analysis is LHC @ home [5], a volunteer computing platform, where people donate idle time on their computers for simulations of physics and beam dynamics. This is another way of creating interest and engagement.

\section{Visits}

One of the most important outreach activities is guided visits [6], giving the opportunity to the public to both see "the real thing" - detectors, accelerators - and to interact with scientists. CERN has more than ten visit sites, which include all LHC experiments. Last year CERN welcomed 150000 visitors from 95 countries, schools in their majority, whereas the demand is a factor of three higher. In addition to official CERN visits, each experiment also organises private tours, usually booked through the secretariat, with collaboration members acting as volunteer guides. They all have visitor centres at the surface which include detector items, posters and videos; and when LHC is not running, they allow underground visits, which is what really fascinates the public.

\section{Virtual Visits}

In an effort to reach remote audiences, unable to visit CERN due to geographical or economical limitations, or simply as an exciting addition to local events, virtual visits were initiated by ATLAS and CMS and adopted by other experiments. Each experiment has its own method of booking and conducting the virtual visits [7-9], with dedicated installations in the control rooms or with mobile devices; they all have a common goal: to give to remote visitors, mainly students, a glimpse of CERN and the experiments, and to offer them the opportunity to interact with scientists. Thousands of people in many countries have visited the LHC experiments virtually in the last years. During the COVID19 pandemic, with all real visits cancelled, virtual visits are a nice substitute.

\section{Open Days and other Events}

In 2019 the CERN Open Days was the most prominent outreach event of the year, for CERN and also for the LHC experiments. During the weekend 14-15 September 2019 more than 75000 
people visited CERN. Many thousands had the chance to visit the LHC experiments' caverns, guided by hundreds of enthusiastic volunteers. In addition to the underground visits a variety of other activities were organised at each LHC site, including talks, demonstrations of simple physics experiments, building detectors out of wooden blocks, lego or cardboard, making proton cookies, listening to the music of cosmic rays and many more.

A variety of other events are regularly organised by CERN, for the local communities but also attracting people from far away, in which the LHC experiments have a strong involvement and participation. During European Researchers' Night, on the last Friday in September, the LHC experiments have offered visits to the control rooms and virtual visits for remote audiences. The CERN and University of Geneva programme "Dans la Peau d'un Chercheur" [10, 11], addressed to elementary school children with the aim to introduce them to the concept of research and inquiry, has been supported with visits to experiments and interviews of scientists. In the last four years female physicists and engineers, many from the LHC experiments, have been giving talks in Swiss and French schools, on the occasion of the International Day for Women and Girls in Science (11 February) [12], in order to serve as role models, against stereotypes that science is for males only.

\section{Online Live Events}

Using the different online platforms available, live events are also organised occasionally, giving the opportunity to wider audiences, practically all over the world, to get first-hand information from the experiments' experts and to ask questions. Some years ago a series of Google Hangouts had taken place, many of them explaining the physics at the LHC [13]. Another format was AMA (Ask Me Anything), originally using the Reddit platform. The preferred method lately is "Facebook Live" events. With the invaluable help of the CERN Audiovisual Service during the last year such events from the experiments' caverns have taken place. It is worth mentioning that the first ever 360 Facebook Live from CERN, from the CMS cavern [14], in October 2019, was the 2020 official honoree of the Webby Awards from the International Academy of Digital Arts and Sciences. Online live events, organised during the period of the COVID19 pandemic, allow to keep alive the interest of the public.

\section{Public Web Sites and Social Media}

The public web sites maintained by the experiments (see Appendix for the URL links), in most cases based on the Drupal content management system which is the CERN standard, are the reference point for information and resources. In addition to static information about detectors and physics, updates are often posted, such as news from detector upgrades and physics briefings.

Different social media platforms allow us to reach audiences of all ages and interests. All experiments use Facebook, Twitter, Instagram (see Appendix for the URL links) to post news and maintain interest and have thousands of followers. Instagram stories, addressing mainly young audiences, seem to become more and more important these days.

Videos are a powerful tool for outreach. All LHC experiments have their Youtube channels (see Appendix), where they post videos with interviews, explanations of physics results or filming 
of detectors. A new series of videos posted on the CERN Youtube channel recently explains for the layman, in three episodes, the process leading to the discovery of the Higgs [15-17].

\section{Arts and Science}

CERN has a varied arts programme [18] involving artists in residence - winners of sponsored competitions - and guest artists, hosted at CERN for a short visit. In this context there is a lot of interaction between scientists and artists, including visits of the experiments, interviews and scientists acting as mentors. This exchange is beneficial for both parts; it provides inspiration to artists and it is stimulating and enriching for us.

Projects such as Art \& Science across Italy [19] and Cultural Collisions by the ORIGIN network [20] are a nice example of connecting science and art. Such projects address mainly school children and involve seminars in schools or universities, visits of museums / laboratories, then workshops by scientists and artists followed by science-inspired artistic creations by students and exhibitions of the art works. The ORIGIN network, in which all LHC experiments participate, also organises outreach events in parallel to conferences, for instance exhibitions and virtual visits, as was the case at LHCP2019 in Puebla, Mexico. Another example of connecting people through science and art was presented at this conference, Ref. [21].

Trying to reach different audiences, LHC physicists organised special sessions on the Physics of Music and the Music of Physics at the Montreux Jazz Festival. Another example of connecting music and physics was the production, for CERN's 60th anniversary, of the LHC Chamber Music video [22]: 6 physicists, inside the underground caverns, played different instruments, based on sonification of data from the LHC experiments.

Big music festivals often look for something different in their programme: science pavillions with experiments and talks were organised at the WOMAD (World Of Music, Art and Dance) festival in 2016. Given the great success, such science programmes were adopted by WOMAD in the following years [23] and also by other festivals such as Moogfest (music, art and technology), the Pohoda festival in Slovakia and the Roskilde festival in Denmark [24]. The feedback from the participants has been very enthusiastic.

\section{Conclusion}

A lot of progress in the outreach efforts of the LHC collaborations has happened in the last years. The huge potential of the variety of online platforms allows to reach audiences across the globe; it also offers the unique possibility for keeping contact with the public in the COVID era. By going to new venues such as music festivals we bring the wonders of science to an even wider public. It is up to us, all members of our community, to make the best possible use of all tools at our disposal. It is important also to encourage others to become active in outreach. And let us not forget that sharing with the public is rewarding and fulfilling; and, as Victor Weisskopf wrote [25], popularisation of science is a potent antidote to overspecialisation, brings out what is significant in current research and makes science a more integral part of the culture of today. 


\section{Appendix}

Links to the public web pages of the four big LHC experiments:

http://alice.web.cern.ch

http://atlas.cern/

https://cms.cern

https://lhcb-public.web.cern.ch

Links to the facebook, twitter and instagram accounts of the four big LHC experiments:

https://www.facebook.com/ALICE.EXPERIMENT/

https://www.facebook.com/ATLASexperiment/

https://www.facebook.com/CMSexperiment/

https://www.facebook.com/LHCbexperiment/

https://www.twitter.com/ALICEexperiment/

https://www.twitter.com/ATLASexperiment/

https://www.twitter.com/CMSexperiment/

https://www.twitter.com/LHCbexperiment/

https://www.instagram.com/alice_experiment/

https://www.instagram.com/atlasexperiment/

https://www.instagram.com/cmsexperiment/

https://www.instagram.com/lhcbexperiment/

Youtube channels of the LHC collaborations:

https://www.youtube.com/c/aliceexperiment

https://www.youtube.com/c/cmsexperiment

https://www.youtube.com/user/TheATLASexperiment

\section{References}

[1] http://ippog.org

[2] http://physicsmasterclasses.org

[3] http://opendata.cern.ch

[4] LHC Open Data for the world to see, presentation by Meirin Oan Evans, this conference

[5] https://hcathome.web.cern.ch

[6] https://visit.cern

[7] https://atlasvirtualvisit.web.cern.ch

[8] https://cms.cern/interact-with-cms/virtual-visits

[9] https://indico.cern.ch/category/10137/

[10] https://voisins.cern/fr/950-pupils-scientists-shoes 
[11] https://www.unige.ch/fapse/dlpc/

[12] https://www.un.org/en/observances/women-and-girls-in-science-day/

[13] https://www.youtube.com/playlist?list=PLAk-9e5KQYEroNUYrWHAANKJL3LDz9-L_

[14] https://www.youtube.com/watch?v=fU0ujGWbeQ0\&t=157s

[15] https://www.youtube.com/watch?v=so2nCu2Jkbc

[16] https://www.youtube.com/watch?v=pW4LTunlXS4

[17] https://www.youtube.com/watch?v=8-WFBGCvv-w

[18] https://arts.cern

[19] https://artandscience.infn.it

[20] http://originnetwork.web.cern.ch

[21] Science and art for connecting people in south-east Europe, presentation by Ivica Puljak, this conference

[22] https://www.youtube.com/watch?v=gPmQcviT-R4

[23] https://home.cern/news/news/knowledge-sharing/cern-womad-worlds-festival

[24] https://www.roskilde-festival.dk/en/years/2019/acts/cern-and-the-niels-bohr-institute/

[25] Science, vol. 176(1972) 